[13] C. F. Harford-Cross, A. B. Carmichael, F. K. Allan, P. A. England, D. A. Rouch and L-L. Wong, Protein Eng. 13 (2000), 121-128.

[14] J. Kazlauskaite, A. C. G. Westlake, L-L. Wong and H. A. O. Hill, J. Chem. Soc. Chem Comm., (1996), 2189-2190.

[15] J. J. Davis, D. Djuricic, K. K. W. Lo, E. M. K. Wallace, L-L. Wong and H. A. O. Hill, Faraday Discuss, 16, (2000), 15-22.

[16] J. Kazlauskaite, H. A. O. Hill, P. Wilkins and H. Dalton, Eur. J. Biochem. 241, (1996), 552 556; Y. Astier, S. Bulendra, H. Dalton and H. A. O. Hill, to be submitted.

[17] J. J. Davis, H. A. O. Hill, A. Kurz, C. Jacob, W. Maret and B. L. Vallee, PhysChemComm, 1, (1998), 20-26.

[18] R. Mukhopadhyay, J. J. Davis, P. Kyritsis, H. A. O. Hill and J. Meyer, J. Inorg. Biochem. 78, (2000), 251-254

[19] A. E. G. Cass, G. Davis, G. D. Francis, H. A. O. Hill, W. J. Aston, I. J. Higgins, E.V. Plotkin, L. D. L. Scott and A. P. F. Turner, Anal. Chem. 56, (1984), 667-671.

[20] M. Kudera, A. Aithen, Li Jiang, S. Kaneko, H. A. O. Hill, P. J. Dobson, P. A. Leigh and W. W. McIntire, J. Electroanal. Chem., 495, (2000), 36-4

\section{WHAT CAN ELECTROCHEMISTRY DO FOR THE ENVIRONMENT?}

J.González-García, V. García, J. Iniesta, V. Montiel, A. Aldaz*

Departamento de Quimica-Fisica. Universidad de Alicante. Apdo. 99. 03080 Alicante. España E-mail: aldaz@ua.es.

\section{Abstract.}

Electrochemistry offers many methods for decreasing the contamination produced by different industrial processes. Among these, Electrochemical Synthesis and Electrochemical Degradation of Organic Compounds in wastewater are two of the more promising ones. There is no doubt that the best way to avoid pollution is to eliminate the process that produces it and in this way very polluting chemical syntheses can be substituted by more cleaner electrochemical processes. As an example of this, the electrochemical synthesis of L-cysteine derivatives from L-cystine will be described. As far as the second method is concerned, the electrochemical degradation of wastewater containing organics appears to be very effective when the wastewater can not be biologically treated and when the oxidation is only carried out until the wastewater can be treated by biological methods. Details of the procedure followed for the development of a pre-industrial process will be shown for the treatment of industrial wastewater containing phenol.

Keywords:L-cysteine, L-cystine; electrochemical synthesis; electrochemical degradation; environmental electrochemistry.

Introducción.

La contaminación medioambiental es uno de los problemas más preocupantes de nuestro desarrollo actual y para evitarla cada día se endurece la legislación sobre vertidos industriales, sean estos sólidos, líquidos o gaseosos. A la Industria Química se le acusa de ser una de las fuentes más importantes de contaminación medioambiental y esto crea una mala imagen social que se extiende no solo a la Química sino también a las áreas con ella relacionadas, olvidando, en muchos casos, que existen multitud de métodos químicos para tratar efluentes industriales de cualquier origen y convertirlos en inocuos. 
No cabe duda que uno de los mejores métodos para disminuir el problema causado por la contaminación es no generarla, por lo que el cambio de procesos, sean estos químicos o de cualquier naturaleza, por otros mas amigos del Medio Ambiente, más verdes en el argot actual, es uno de los mejores procedimientos para conservar intacto nuestro entorno. Sin embargo, es a veces imposible cambiar un proceso, por ejemplo químico, por otro menos polucionante, bien sea porque la calidad del producto final no es la adecuada o bien por que el nuevo proceso es mucho más costoso que el existente. No deberia olvidarse que la sustitución de un proceso que funciona y que la Industria conoce perfectamente, por otro, quizás mejor pero de comportamiento desconocido a largo tiempo, no suele ser del agrado de los industriales, a no ser que el proceso mejore en mucho al existente o la legislación medioambiental hagan imposible el viejo proceso por los costes implicados en el tratamiento de los efluentes.

El tratamiento de los residuos industriales es hoy en día un tema en auge en investigación ya que es necesario descubrir y optimizar nuevos procesos de tratamiento de efluentes que logren la detoxificación del residuo a costes asumibles por la Industria. Entre estos nuevos procedimientos destacan los derivados de la tecnología electroquímica que, por su gran versatilidad, se presentan como métodos a tener en cuenta en el tratamiento de numerosos efluentes. Así, la figura 1 muestra un esquema, no exhaustivo, de las posibilidades de la Electroquímica en el tratamiento de efluentes industriales.

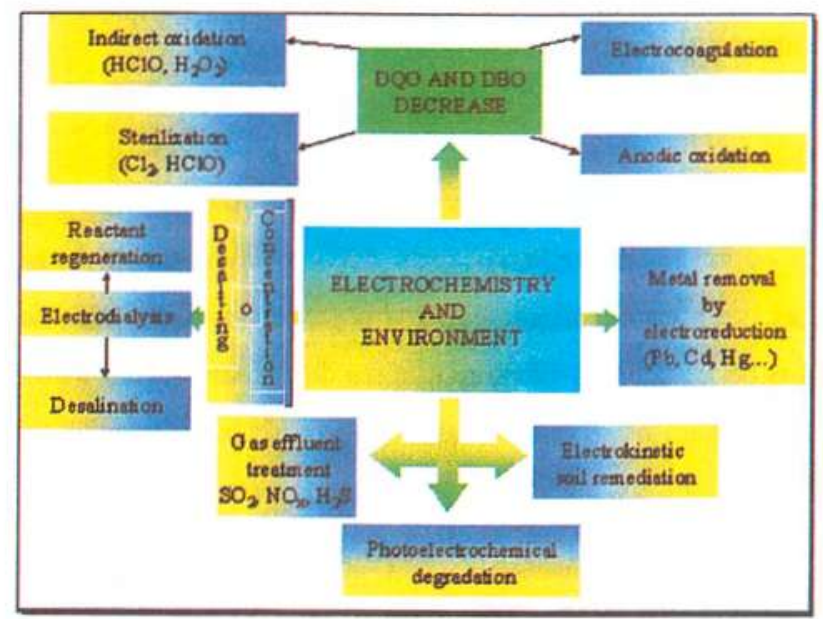

Figura 1.-Posibilidades de la Electroquímica en el tratamiento de efluentes
A lo largo de estas líneas intentaremos demostrar las posibilidades que posee la Electroquímica como tecnología capaz de i)desarrollar nuevos y menos contaminantes procesos de síntesis química y ii) desarrollar nuevos procedimientos para el tratamiento de vertidos industriales. Por ello, trataremos el desarrollo de un proceso de sintesis electroquímica de derivados de la L-cisteína, desde la escala de laboratorio a la planta piloto industrial, y de un proceso de destrucción de materia orgánica de un efluente procedente de una industria que contiene una elevada concentración de fenoles y no puede ser tratado por los métodos biológicos ordinarios.

\section{Síntesis electroquímica de productos farmacéuticos.}

Tanto la L-cisteína como sus derivados S-carboximetil L-cisteína y $\mathrm{N}$-acetil Lcisteína, son productos farmacéuticos utilizados en el tratamiento de bronquitis y resfriados, o como agentes protectores hepáticos ( $\mathrm{N}$-acetil L-cisteina). Una de las síntesis clásicas más común de L-cisteína es su obtención por reducción con $\mathrm{Zn}$ en polvo de L-cistina. Una vez obtenida, la L-cisteína se puede transformar en Scarboximetil L-cisteína, por ataque con monocloroacetato sódico, o en $\mathrm{N}$-acetil Lcisteína por acetilación con anhídrido acético (aunque también puede obtenerse a partir de la reducción de la NN' diacetil L-cistina). La figura 2 muestra un esquema general para la obtención de estos productos.

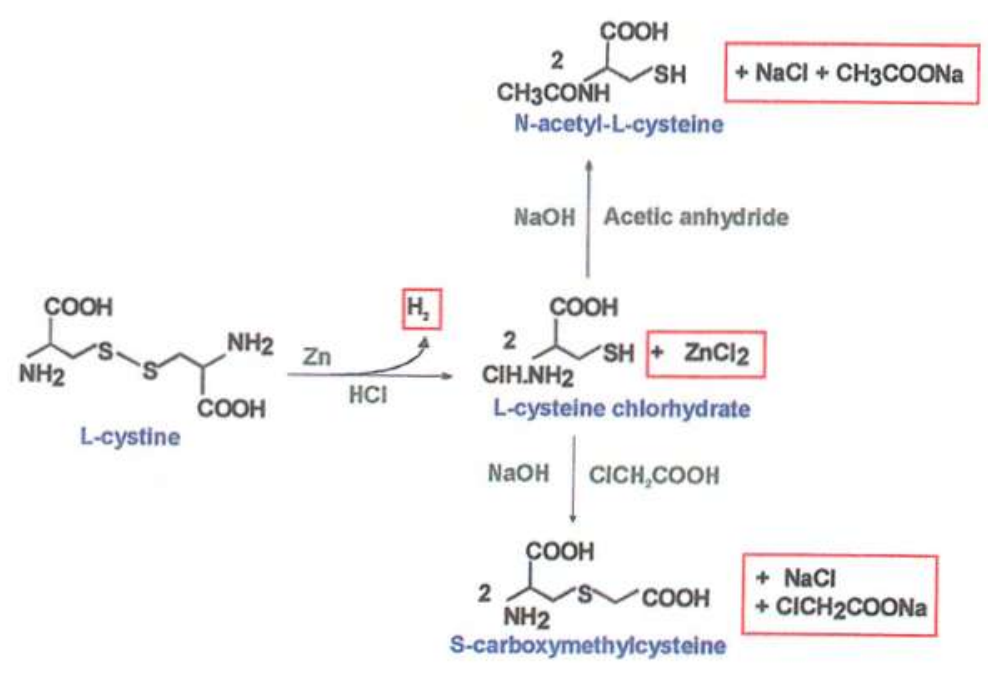

Figura 2.-Rutas químicas para la obtención de los derivados de la L-cisteina. 
Los principales inconvenientes de la síntesis química son: i) Tiene un alto impacto medioambiental ya que genera un efluente altamente concentrado en $\mathrm{Zn}^{2+}$; ii) Consume una cantidad muy elevada, mayor que la estequiométrica, de $\mathrm{Zn}$ por corrosión de este; iii) Es una reacción fuertemente exotérmica con una gran producción de $\mathrm{H}_{2}$ por lo que exige fuertes medidas de seguridad; iv) La cristalización del aminoácido se realiza en un medio fuertemente salino con una elevada concentración metálica, por lo que el rendimiento de la cristalización no es muy bueno ni tampoco lo es la pureza del producto final que debe ser por tanto recristalizado.

\section{Síntesis electroquímica.}

La ruptura catódica del enlace S-S de la L-cistina y de la N,Ndiacetil L-cistina es una reacción electroquímica sencilla que se puede realizar sobre cátodos con elevado sobrevoltaje de hidrógeno con gran facilidad y rendimiento [1-17] por lo que se puede fácilmente sustituir la reducción química con polvo de $\mathrm{Zn}$ por la reducción electroquímica.

Dada la escasa penetración que la tecnología electroquímica tiene en la Industria Química y los recelos que esta tecnología despierta, se decidió realizar un trabajo de Investigación y Desarrollo que abarcara desde los estudios iniciales voltamétricos al proceso de optimización a escala planta piloto industrial. Lo que a continuación sigue es un resumen de las diferentes etapas desarrolladas, de la metodología seguida y de los logros obtenidos en la síntesis a escala industrial de S-carboximetil L-cisteína de la que se han sintetizado en la planta piloto industrial electroquímica de la Universidad de Alicante un total de $14.000 \mathrm{Kg}$ a lo largo de diez meses de trabajo.

\section{1.- Estudio de Laboratorio}

Como primera etapa se estudió el comportamiento voltamétrico de la reducción de L-cistina en diferentes medios, para averiguar si el proceso podía ser realizado, primero de forma electroquímica, y segundo, si poseía las características necesarias para proceder a su escalado industrial.

El interés, por tanto, de este estudio se puede resumir en los siguientes aspectos:
A) Características globales del proceso: i) Electrocatálisis: materiales electródicos convenientes como cátodos; ii) Tipo de procesos que controlan la corriente: difusión, transferencia electrónica; iii) Reversibilidad electroquímica: necesidad o no de un separador entre anolito y catolito; iv) Interferencias con otras posibles reacciones; v) Identificación de productos.

B) Técnicas empleadas: i) Curvas I-E con electrodo rotatorio; ii) Voltametría; iii) Electrolisis a potencial y corriente controladas.

Electrochemical reduction of

CYSTINE (Voltammetric study)

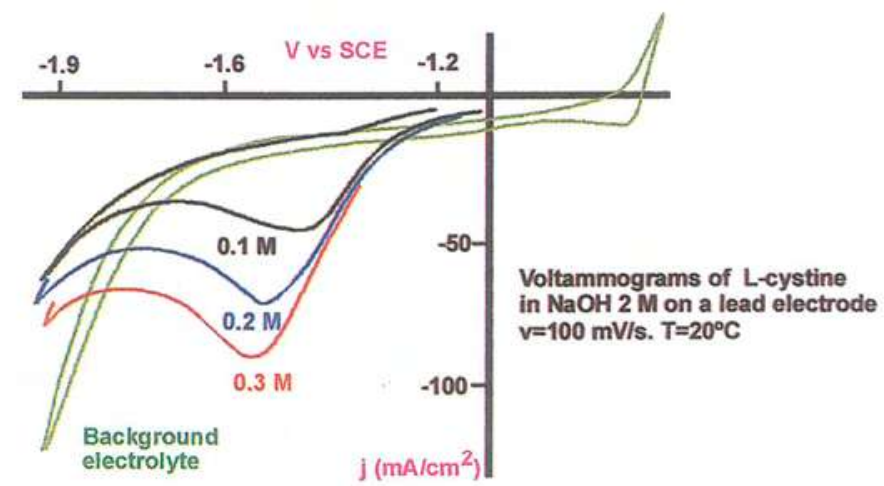

Figura 3.- Voltametría de L-cistina en $\mathrm{NaOH} 4 \mathrm{M}$ sobre un electrodo de plomo. v=100 $\mathrm{mV} \mathrm{s} \mathrm{s}^{-1} . \mathrm{T}=20^{\circ} \mathrm{C}$

La figura 3 muestra algunas de las curvas voltamétricas obtenidas. Se ve claramente que el proceso de reducción es factible si se emplea un electrodo de elevado sobrevoltaje, tal y como es el plomo, ya que la reducción de la L-cistina se produce a potenciales menos negativos que la reducción del disolvente $\mathrm{H}_{2} \mathrm{O}$. Se debe indicar que los productos utilizados para realizar estas curvas fueron de calidad industrial, muy inferior a la típica empleada en este tipo de estudios. 


\section{Electrochemical reduction of}

CYSTINE (Laboratory reactor)

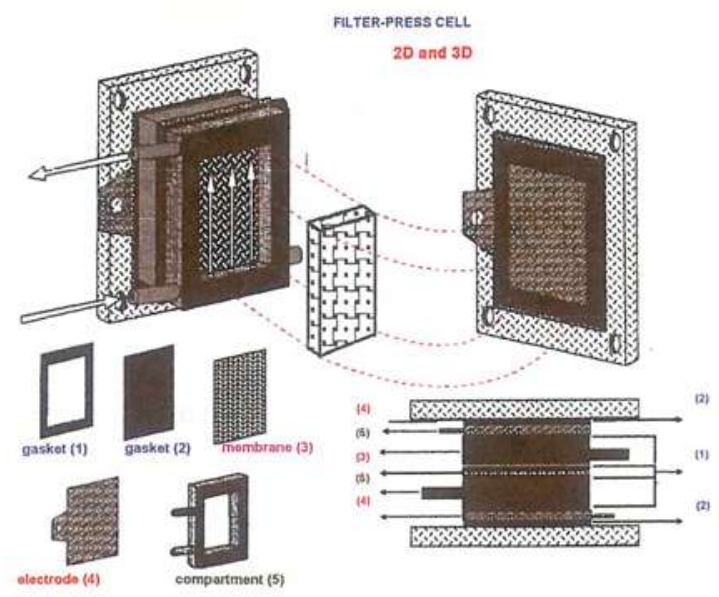

Figura 4.- Despiece de una célula filtro-prensa de laboratorio $\left(A=20 \mathrm{~cm}^{2}\right)$

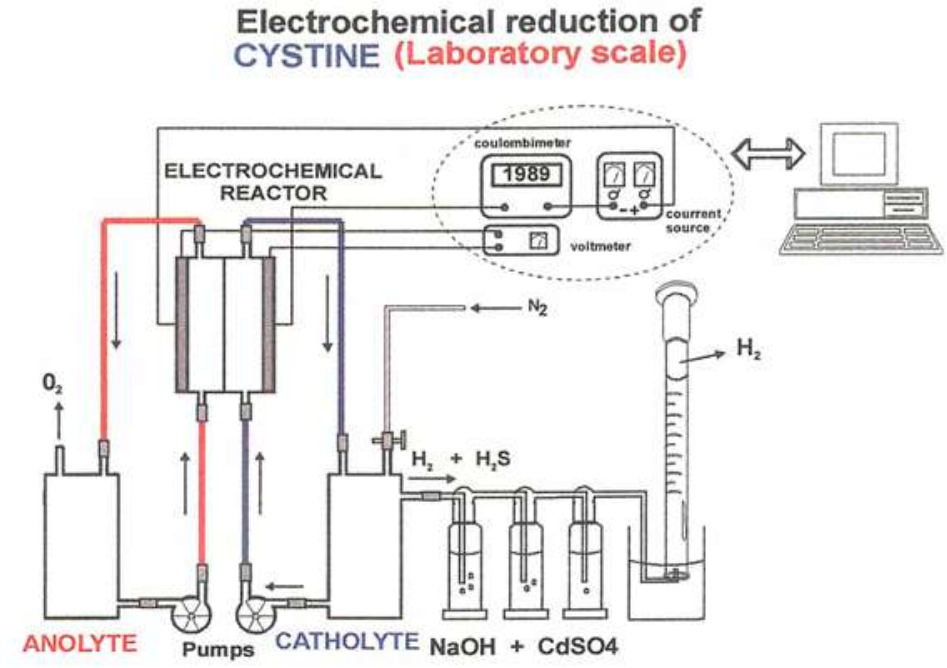

Figura 5.- Dispositivo experimental empleado para el estudio en laboratorio.
Las sintesis para la identificación del producto final y para estudiar su calidad se realizaron en reactores filtro-prensa con áreas entre 20 y $60 \mathrm{~cm}^{2}$. Como reacción anódica se empleó la formación de oxígeno a partir de disoluciones de $\mathrm{Na}_{2} \mathrm{SO}_{4}$ o $\mathrm{H}_{2} \mathrm{SO}_{4}$ y como membrana separadora una catiónica, Nafion. La figura 4 muestra un esquema de una de las células y la figura 5 el dispositivo experimental empleado. La figura 6 muestra un esquema del proceso de reducción de la L-cistina, mientras que la figura 7 muestra la sintesis de S-carboximetil L-cisteína.

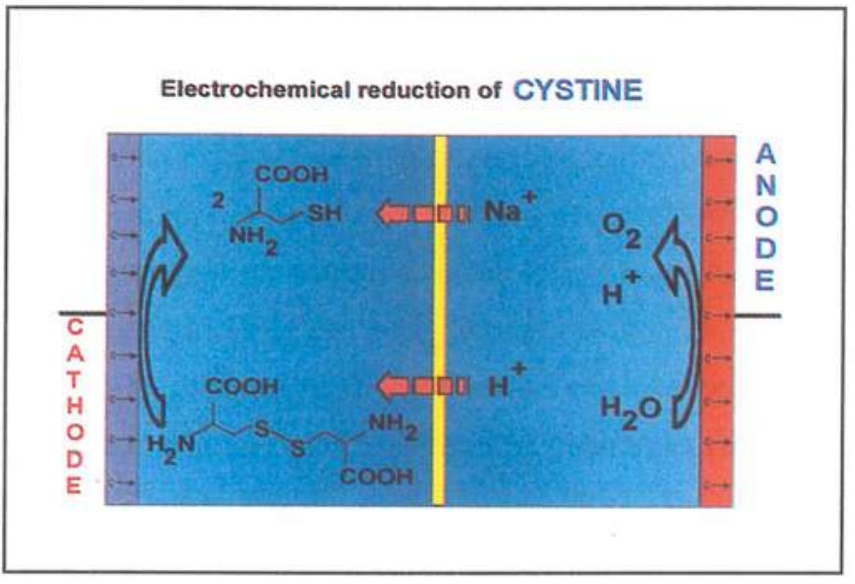

Figura 6.- Proceso de reducción electroquímica de L-cistina

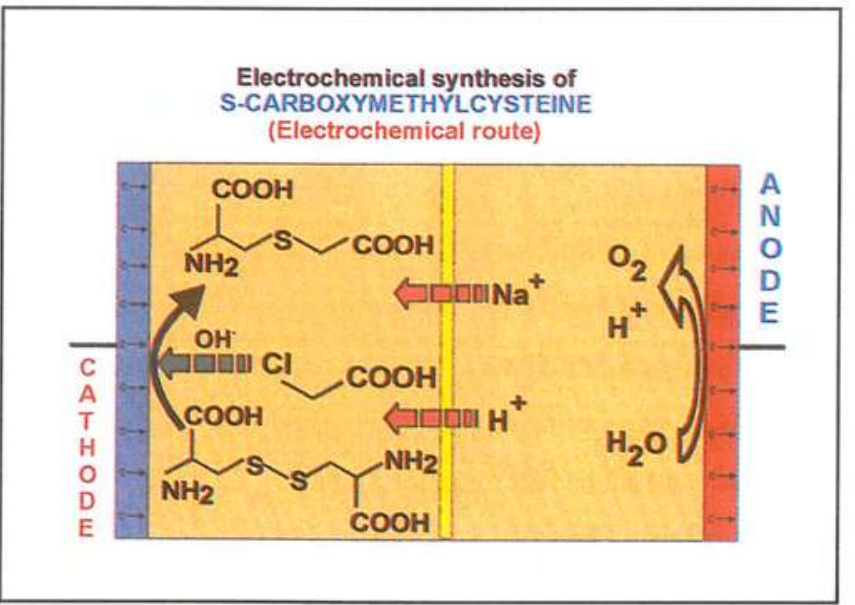

Figura 7. Proceso de síntesis electroquímica de S-carboximetil-L-cisteína 
Las conclusiones que deseamos obtener al llevar a cabo este estudio deben proporcionar información clara y precisa sobre: i) Material catódico a emplear; ii) Valores de corriente que pueden ser empleadas en los procesos de sintesis (una vez conocida la constante de transporte de materia del reactor electroquímico que queremos emplear); iii) Tipo de célula a emplear - dividida, es decir con dos compartimentos separados por una membrana, o no-dividida, un compartimento; iv) Identificación y caracterización del producto obtenido, su pureza; v) Posibilidad de llevar a cabo la sintesis electroquímica.

\section{Estudio a escala pre-industrial}

El interés de este estudio es realizar un salto de escala en el proceso de sintesis y a la vez, profundizar en el conocimiento del proceso, especialmente en los aspectos relacionados con la estabilidad de los electrodos, ánodo y cátodo, y en el rendimiento pureza del producto. Para ello empleamos un reactor electroquímico de mayor tamaño, área electródica $200 \mathrm{~cm}^{2}$, y se aumentó la duración de las experiencias de sintesis electroquímica a tiempos superiores a las 100 horas de proceso.

Los parámetros estudiados en esta parte del desarrollo fueron: i) Selección de electrodos y electrolitos, ii) Comportamiento de los materiales electródicos, iii) Rendimiento de la reacción en materia y corriente, iv) Selección definitiva de la reacción anódica que se va a emplear, v) Influencia de la concentración inicial de Lcistina y del $\mathrm{pH}$ en el rendimiento de la síntesis, vi) Pureza del producto final, vii) Coste eléctrico del producto, $\mathrm{kWh} / \mathrm{kg}$, (poco importante para productos farmacéuticos), viii) Productividad del reactor, $\mathrm{kg} / \mathrm{m}^{2}$.día para una determinada densidad de corriente, ix) Conclusiones preliminares acerca de la posibilidad industrial de realizar la sintesis y de sus parámetros económicos.

La figura 8 muestra la influencia del tipo de electrolito sobre el rendimiento de la sintesis electroquimica empleando un cátodo de plomo, junto con los datos de coste energético y producción -esta última para distintos porcentajes de carga pasada-.

En este punto, y para aumentar la productividad y rendimiento en materia del proceso, se decidió estudiar el comportamiento de los llamados electrodos tridimensionales que ofrecen, frente a los clásicos bidimensionales, un área electródica muy superior, por lo que, empleando la misma densidad de corriente, y por tanto conservando la selectividad del proceso, permiten la utilización de corrientes muy superiores, lo que aumenta extraordinariamente la productividad del reactor electroquímico. La figura 9 muestra los resultados obtenidos empleando como electrodo tridimensional un fieltro de carbón. Dado el gran aumento de productividad se decidió, a partir de este momento, continuar el trabajo empleando este tipo de electrodos.

\section{Electrochemical reduction of}

CYSTINE (Influence of the type of electrolyte)

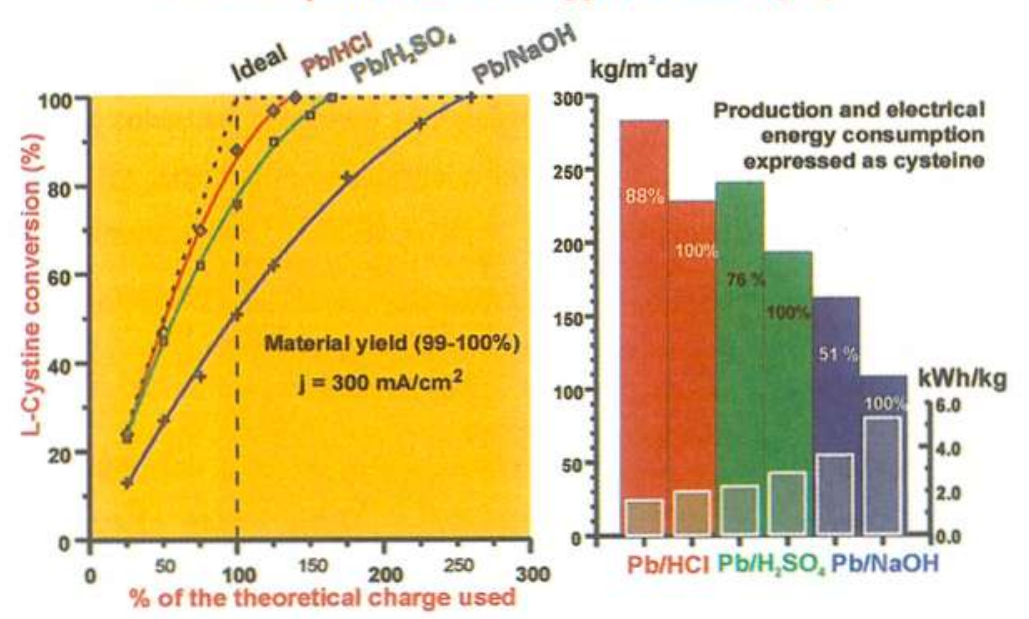

Figura 8.- Influencia del electrolito en la conversión, producción y consumo energético del proceso.

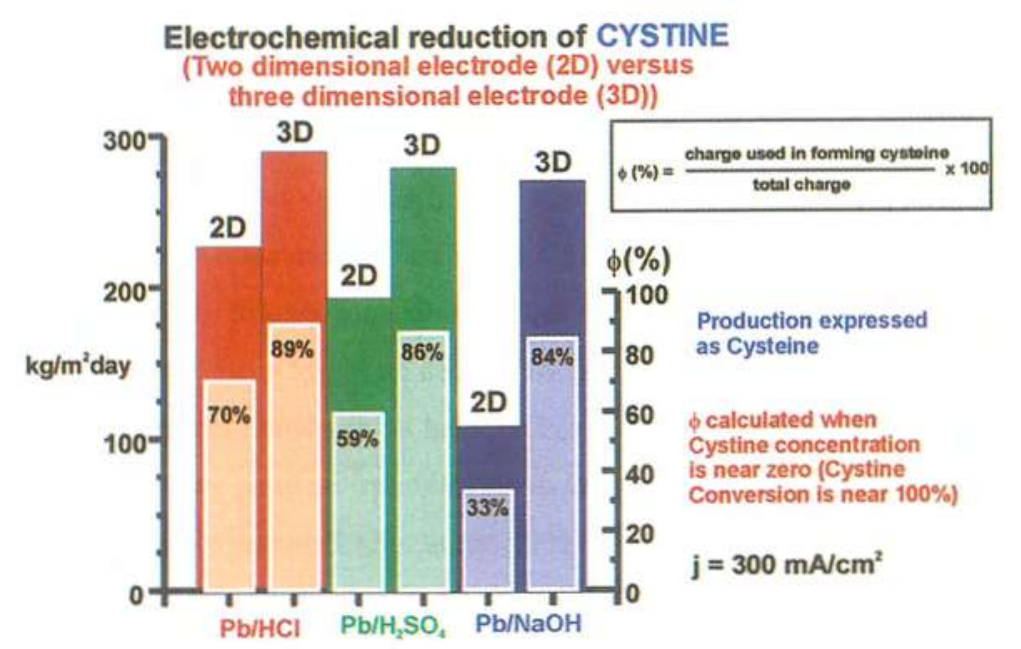

Figura 9.- Comparación de producción y eficiencia en corriente obtenidos para electrodos bidimensionales vs tridimensionales. 


\section{Estudio a escala industrial.}

Para llevar a cabo este estudio se realiza un nuevo cambio de escala, aumentado el área electródica unidad hasta $1000-3000 \mathrm{~cm}^{2}$ y empleando reactores filtro-prensa de tipo industrial. Durante este estudio se deben obtener conclusiones definitivas sobre los siguientes aspectos: i) Tipo de conexión eléctrica de las células: montaje monopolar o montaje bipolar, ii) Comportamiento de electrodos, membranas y otros componentes del reactor para tiempos de 1000 horas, iii) Calidad del producto final, iv) Problemas causados por la calidad de los productos iniciales, v) Problemas causados por el posible parada del reactor electroquímico y métodos de puesta en marcha, vi) Métodos de limpieza del reactor electroquímico entre paradas, vii) Posibles tratamientos de los efluentes, viii) Economía del proceso, ix) Escritura del libro de proceso, x) Toma de decisiones.

Este estudio se llevo a cabo en la planta piloto industrial del Departamento de Química-Física, siendo los datos más importantes de la planta electroquímica: i) Reactor electroquímico filtro prensa de $1000-3000 \mathrm{~cm}^{2}$ de área, ii) Rectificador de $30 \mathrm{~V}$ $1000^{\mathrm{a}}$ controlado por ordenador, iii) Depósitos de anolito y catolito de $1 \mathrm{~m}^{3}$, iv)Sistemas auxiliares: detectores, sondas $\mathrm{pH}$ y conductividad, bombas dosificadoras etc.

La S-carboximetil L-cisteína puede ser obtenida, como muestra la figura 7, en una sola etapa realizando la reducción en medio básico de la L-cistina en presencia de monocloroacetato sódico que se añade gradualmente a lo largo de la síntesis. Las condiciones experimentales de la síntesis son: i) Cátodo: fieltro de carbón vítreo, ii) Catolito: L-cistina en disolución de $\mathrm{NaOH}$ con una concentración inicial de $0.8-1 \mathrm{M}$ y un $\mathrm{pH}$ 8-13, iii) Anodo: Un ánodo dimensionalmente estable para la formación de oxígeno (DSA-O $\mathrm{O}_{2}$ ), iv) Anolito: disolución acuosa de $\mathrm{Na}_{2} \mathrm{SO}_{4}$, v) Separador: membrana Nafion o Neosepta ambas catiónicas, v) Densidad de corriente: entre 200 y $2000 \mathrm{~A} / \mathrm{m}^{2}$, vi) Reactor: Célula bipolar con un total de $9 \times 2500 \mathrm{~cm}^{2}$ de área, vii) Número de batches y tiempo de electrolisis: 132 batches y 1000 horas, viii) Parámetros económicos: coste 1 $\mathrm{kWh} / \mathrm{kg}$ y producción $190 \mathrm{~kg} / \mathrm{m}^{2}$.día. Producción Total $14000 \mathrm{Kg}$

El producto obtenido cumplía con las características exigidas por la Farmacopea Europea. La figura 10 muestra una vista de la planta piloto industrial del Departamento.

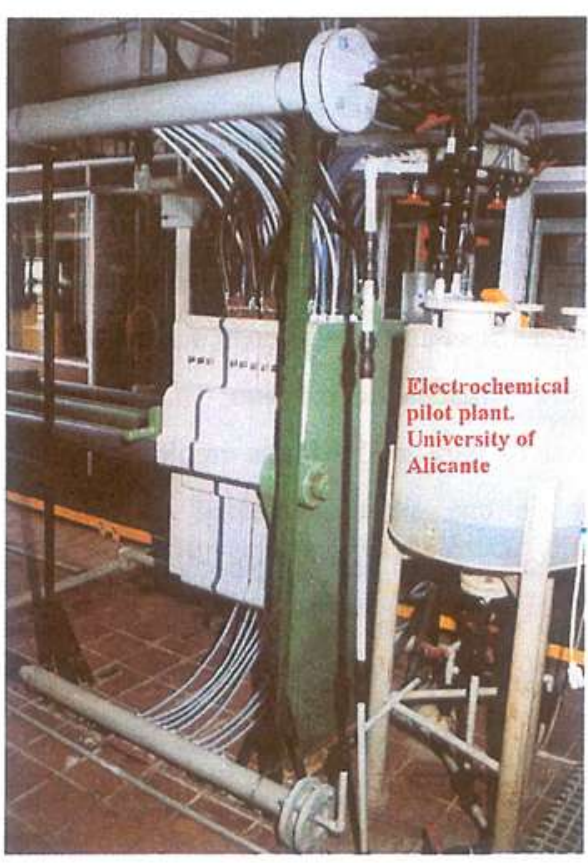

Figura 10.- Detalle de la planta piloto industrial del Departamento de Química Física de la Universidad de Alicante. 


\section{Destrucción de materia orgánica en aguas residuales industriales.}

La eliminación de materia orgánica y de metales pesados, en aguas residuales procedentes de la industria química, puede también realizarse empleando métodos electroquímicos. En este punto cabe advertir que el mejor método para la detoxificación de efluentes es el tratamiento biológico, tanto por su sencillez como por su bajo coste. Solamente cuando las aguas residuales no puedan ser tratadas por estos métodos, bien por contener una elevada demanda de oxígeno, ya sea química, o biológica, o por contener compuestos tóxicos para las bacterias empleadas en el tratamiento, tal como puede ser la presencia de una elevada cantidad de metales pesados, compuestos químicos bactericidas, etc., debe ser empleado el proceso electroquímico de detoxificación. Incluso en estos casos, se debe utilizar la Electroquímica solo hasta obtener unas aguas residuales que puedan ser tratadas biológicamente.

Se debe indicar que en la mineralización de la materia orgánica, es decir, su oxidación total a $\mathrm{CO}_{2}$, se transfiere un número de electrones muy elevado -por ejemplo, la oxidación de una molécula muy sencilla como la de metanol exige 6 electrones-, por lo que la energía eléctrica necesaria para la transformación es muy elevada y hace que el proceso no sea rentable económicamente. Así por ejemplo, aceptando un potencial de electrolisis de $3 \mathrm{~V}$ y un rendimiento en corriente del $100 \%$, el coste de disminuir 1000 ppm de DQO en un agua residual es $0,01 \mathrm{kWh} /$ litro. La destrucción de materia orgánica en las aguas residuales debería realizarse hasta un punto a partir del cual pueda emplearse el tratamiento biológico. Es esta la única posibilidad de utilización de la Electroquímica; intentar obtener un efluente final que cumpla las normas legales sobre efluentes solo por métodos electroquímicos, suele conducir al fracaso cuando se desea desarrollar un proceso industrial. Sin embargo, la eliminación electroquímica de metales pesados, al ser menor el número de electrones intercambiados y las disoluciones más diluidas, suele ser un proceso económicamente viable.

Como ejemplo del desarrollo de un proceso electroquímico de tratamiento de aguas residuales, describiremos los pasos llevados a cabo en el proceso de tratamiento de unas aguas residuales industriales de una empresa textil que contienen una cantidad de fenol muy elevada y que hace inviable el proceso biológico. En este caso, el grado de desarrollo será menor ya que sólo llegaremos a la etapa piloto pre-industrial.

Las etapas del desarrollo del proceso son muy similares a las descritas para el proceso de sintesis, al fin y al cabo se trata de transformar una molécula en otra, pero aquí se debe prestar una especial atención a los parámetros, energía consumida por $\mathrm{m}^{3} \mathrm{de}$ efluente tratado y tamaño del reactor necesario por $\mathrm{m}^{3}$ de efluente tratado, dado que son los que determinarán si el proceso es industrialmente viable.

El objetivo final de este estudio es la obtención de efluente final con las características: i) Contenido final en fenoles $<2 \mathrm{ppm}$, ii) DQO final del efluente $<1000$ ppm, iii) Sólidos totales en suspensión finales $<300 \mathrm{mg} / \mathrm{L}$, iv) $\mathrm{pH}$ final del efluente entre 6 y 9 .

\section{Estudio de laboratorio}

Suele ser tradicional empezar este estudio empleando una agua residual sintética con el componente que estamos interesados en eliminar [19-21]. Si bien se pueden obtener conclusiones interesantes de este estudio, no se debe olvidar que las aguas residuales tienen una composición muy compleja y que es prácticamente imposible, industrialmente, que exista un agua residual en la que sólo haya componente. Por tanto, estos resultados, obtenidos empleando el mismo protocolo que se realiza en la Síntesis Electroquímica y que ha sido mostrado anteriormente, suelen ser de valor muy relativo. Además, no solo la composición de las aguas residuales es muy compleja, sino que cambia, incluso a lo largo del día, en composición y en concentración, entre márgenes muy variables por lo que el proceso desarrollado debe ser capaz de aceptar estas variaciones sin pérdida de eficacia. Como vía de ejemplo, la Tabla I muestra la composición de una serie de muestras provenientes de la empresa textil. En ella se ve que tanto la concentración de fenol como la del ion $\mathrm{Cl}^{-}$(cuya importancia después se indicará) varía en amplios márgenes.

\begin{tabular}{c|cccccc}
\hline Analysis & $\begin{array}{c}\text { Sample } \\
\text { L01 }\end{array}$ & $\begin{array}{c}\text { Sample } \\
\text { L02 }\end{array}$ & $\begin{array}{c}\text { Sample } \\
\text { L03 }\end{array}$ & $\begin{array}{c}\text { Sample } \\
\text { L04 }\end{array}$ & $\begin{array}{c}\text { Sample } \\
\text { L05 }\end{array}$ & $\begin{array}{c}\text { Sample } \\
\text { L06 }\end{array}$ \\
\hline Phenol (ppm) & 115 & 171 & 253 & 42 & 127 & 183 \\
COD (ppm) & 10000 & 12350 & 14800 & 4000 & 6200 & 8000 \\
pH & 8.9 & 8.8 & 9.0 & 9.7 & 10.1 & 10.0 \\
$\begin{array}{c}\text { Conductivity } \\
\left(\mathbf{m S c m}^{-1}\right)\end{array}$ & 11.0 & 11.0 & 15.6 & 9.2 & 10.2 & 9.6 \\
Turbidity (NTU) & 15 & 116 & 308 & 3 & 30 & 125 \\
\hline
\end{tabular}

Tabla 1. Características iniciales más relevantes correspondientes a los muestras enviadas por la empresa. 
Puesto que el proceso de oxidación del agua puede ser competitivo con el de oxidación de la materia orgánica, parece conveniente empezar nuestro estudio empleando un electrodo de $\mathrm{Ti} / \mathrm{PbO}_{2}$, que posee un elevado sobrevoltaje de oxígeno. Puesto que parece demostrado que la generación de radicales $\mathrm{OH}$ es una etapa conveniente en la destrucción orgánica [22] se ha estudiado también el comportamiento de electrodos de $\mathrm{PbO}_{2}$ dopados con Bismuto que se caracterizan por formar radicales $\mathrm{OH}$ más fácilmente que el $\mathrm{PbO}_{2}$ puro.

Los voltagramas de las Figuras 11A y 11B muestran las curvas corriente voltaje en ausencia y presencia de fenol. Se puede comprobar que el fenol se oxida a potenciales muy próximos a los necesarios para el desprendimiento de $\mathrm{O}_{2}$. La disminución de corriente con el ciclado demuestra la existencia de un proceso de inhibición que puede ser debido a la formación de un polímero de fenol oxidado sobre la superficie.

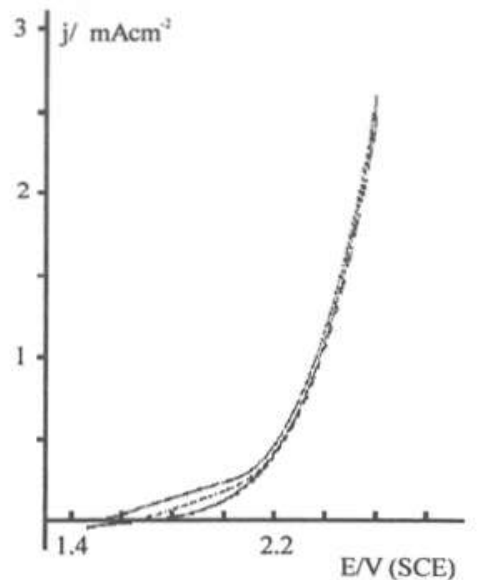

A

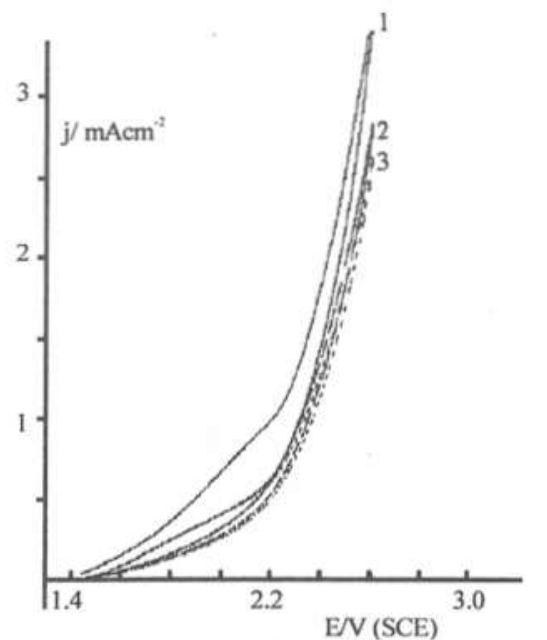

Figura 11. Comportamiento voltamétrico del electrodo $\mathrm{Ti} / \mathrm{PbO}_{2}$ en $\mathrm{H}_{2} \mathrm{SO}_{4} 0.5 \mathrm{M}$. (A) Ausencia de fenol, (B) $10^{-2} \mathrm{M}$ de fenol, tres ciclos. $\mathrm{v}=50 \mathrm{mVs}^{-1}$.

Visto pues, que parece posible la destrucción del fenol por oxidación, se procedió al estudio en célula filtro prensa de un agua sintética conteniendo este compuesto [23]. Puesto que las aguas residuales que se van a tratar contienen $\mathrm{Cl}$, se ha estudiado desde el comienzo, la influencia de la presencia de $\mathrm{Cl}^{\prime}$ en la oxidación del fenol. La Figura 12 muestra que esta presencia es positiva para la oxidación del fenol. El cambio del electrodo de $\mathrm{PbO}_{2}$ por el de $\mathrm{B} / \mathrm{PbO}_{2}$ no evidenció ninguna mejora significativa.

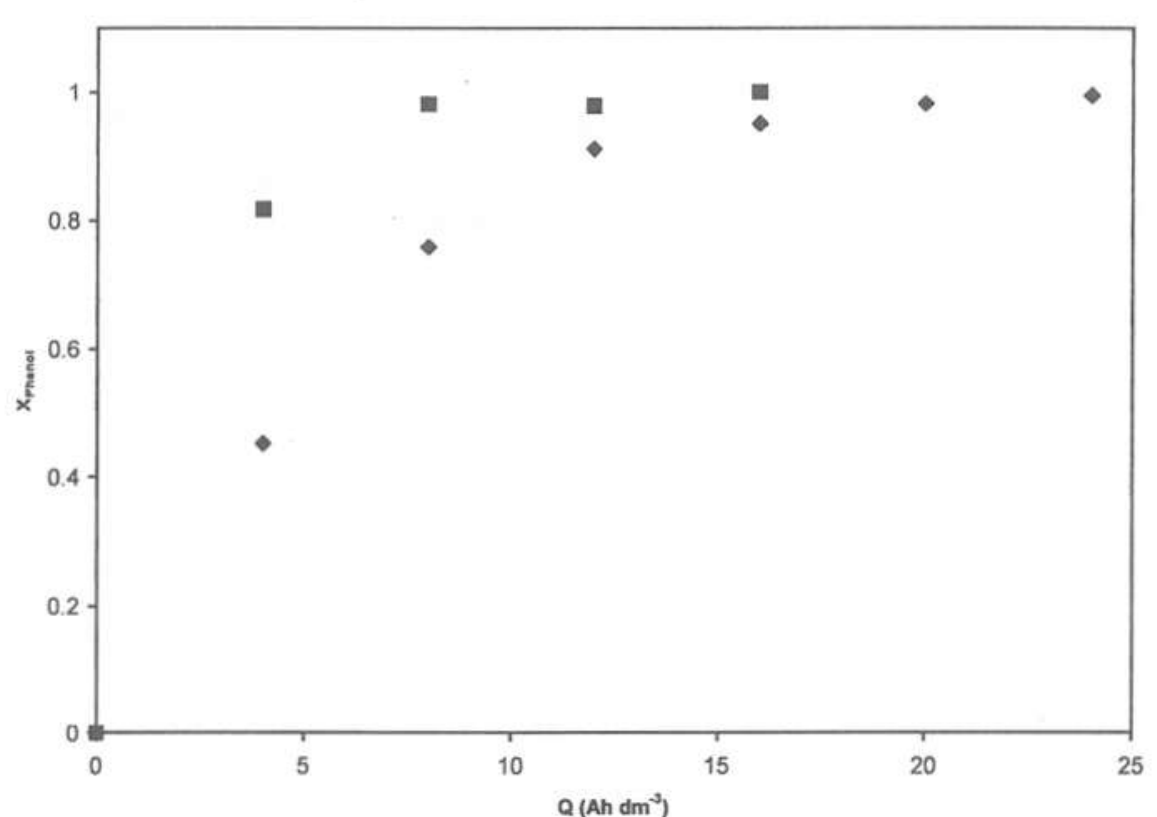

Figura 12. Fracción de conversión del fenol en función de la carga pasada. $\mathrm{T} / \mathrm{PbO}, 0.5$ $\mathrm{M} \mathrm{Na}_{2} \mathrm{SO}_{4}, \mathrm{pH} 12,100 \mathrm{~mA} \mathrm{~cm}{ }^{-2}, 1000 \mathrm{ppm}$ de fenol, $\bullet \sin \mathrm{Cl}^{-}(v)$ con Cl .

Los análisis necesarios para identificación y cuantificación de los diferentes compuestos se llevaron a cabo de acuerdo con los métodos indicados en la Tabla 2. 


\begin{tabular}{l|l}
\hline \multicolumn{1}{c|}{ Parameter } & \multicolumn{1}{c}{ Method } \\
\hline Phenols & 4-aminoantipirine \\
COD & USEPA 604 \\
Halocompound & Colourimetric \\
Gas chromatography with mass spectrometry detector \\
$\mathbf{s}$ & GC//MS \\
Cations & Purge and trap coupled with a GC//MS \\
Anions & Mass spectrometry ICP-MS \\
Gases & Ion exchange chromatography IC \\
\hline
\end{tabular}

Tabla 2. Métodos analíticos utilizados para determinar las características iniciales y finales del efluente.

Para estudiar la oxidación del efluente a escala laboratorio se ha empleado un reactor filtro prensa diseñada en nuestro Departamento $\left(63 \mathrm{~cm}^{2}\right.$ de área geométrica) [24]. Se empleó una disolución de fenol como agua residual y del estudio se determinó: i) Estabilidad de los ánodos, ii) Elección del material catódico, iii) Tipo de célula: dividida o no dividida, iv) Densidades de corriente a emplear; ¿ fija o variable.?, v) Temperatura, $\mathrm{pH}$, tipo de electrolito, vi) Influencia de la presencia o no de iones cloruro, vii) Concentración final de fenol, viii) Demanda Química de Oxígeno (DQO) final, ix) Composición de purgables y fase líquida, $\mathrm{x}$ ) Composición de la fase gaseosa, xi) Comportamiento de los electrodos, xii) Uso de membranas o separadores, xiii) Precipitación de sólidos y formación de espumas.

Los resultados obtenidos fueron muy satisfactorios, obteniéndose concentraciones finales de fenol menores que $5 \mathrm{ppm}$. La presencia de ion $\mathrm{Cl}^{2}$ mejoraba sensiblemente los resultados. Las condiciones más favorables obtenidas fueron:

Ánodo: $\mathrm{Ti} / \mathrm{PbO}_{2}$.

Cátodo: Acero inoxidable.

Separador: Membrana catiónica

Catolito: Ácido sulfúrico.

Anolito: $\mathrm{pH}$ alcalino (11-12) Fenol 1000ppm.

Temperatura $40^{\circ} \mathrm{C}$.

Densidad de corriente: $100 \mathrm{~mA} \mathrm{~cm}^{-2}$.

Carga pasada: $150 \%$ respecto a la $\mathrm{DQO}\left(\mathrm{mgO}_{2} / \mathrm{L}\right)$ inicial.
Una vez comprobado que el fenol puede ser eliminado, empleando el mismo dispositivo se estudio el comportamiento de aguas residuales reales a diferentes $\mathrm{pH}$ cuyas características se han mostrado en la Tabla 1 Es de destacar la gran variabilidad de la concentración de fenol con concentraciones que van de $40 \mathrm{ppm}$ a $300 \mathrm{ppm}$. Los valores de DQO presentan también grandes oscilaciones, entre 4000 y $15000 \mathrm{ppm}$. La concentración de $\mathrm{Cl}^{-}$es muy elevada -de 4 a $7 \mathrm{~g} / \mathrm{dm}^{3}$ - al igual que la de $\mathrm{Ca}^{2+}$, de 2 a 4 $\mathrm{g} / \mathrm{dm}^{3}$.

La Tabla 3 muestra que el tratamiento electroquímico, al 100\% de carga pasada referida a la DQO total, es capaz de lograr un agua residual con la concentración final requerida de fenol.

\begin{tabular}{c|cccc}
\hline FINAL & pH 2 & pH 5-7 & pH 11-12 & pH 12-7 \\
\hline Phenol conc (ppm) & $33(91 \%)$ & $12(97 \%)$ & $1(99.8 \%)$ & $2.5(99 \%)$ \\
COD(decrease \%) & 62 & 57 & 48 & 58 \\
Colour & colourless & colourless & colourless & colourless \\
\hline
\end{tabular}

Tabla 3. Análisis final del agua residual después del tratamiento electroquímico.

Sin embargo, la presencia de compuestos orgánicos hace indeseable la generación de $\mathrm{Cl}_{2}$ ya que este es un fuerte agente clorante por lo que podrían generar fácilmente cloroderivados que pueden ser más peligrosos que los productos iniciales, aunque estuvieran en concentraciones inferiores. El empleo de un $\mathrm{pH}$ básico produce la hidrólisis de $\mathrm{Cl}_{2}$ a hipoclorito, que posee una menor capacidad de cloración que el $\mathrm{Cl}_{2}$ conservando un fuerte carácter oxidante, por lo que se decidió continuar el estudio en este medio.

La ausencia de un separador entre anolito y catolito provoca la formación de espumas que afectan al proceso de electrólisis y al transporte de materia en la célula. Se decidió por tanto, emplear una membrana de intercambio iónico para evitar esta formación y realizar la oxidación anódica a pH básico. 
Dado que el efluente final debiera tener un pH en el entorno de 6-8 de acuerdo con la legislación, es posible utilizar tres métodos diferentes de electrolisis, para llevar a cabo la oxidación:

Método 1: Uso de una membrana catiónica. Electrólisis a pH entre 11 y 12 (adición de $\mathrm{NaOH}$ para controlar el $\mathrm{pH}$ ) para evitar la formación de cloro y adición final de un ácido para llevar el $\mathrm{pH}$ hasta 7.

Método 2: Uso de una membrana catiónica. Electrólisis a pH entre 11 y 12 (adición de $\mathrm{NaOH}$ para controlar el $\mathrm{pH}$ ) para evitar la formación de cloro. En la última parte de la electrolisis cesa la adición de $\mathrm{NaOH}$ para que el $\mathrm{pH}$ disminuya hasta 6-8 $\mathrm{pH}$, valor requerido para el vertido del efluente.

Método 3: La electrolisis se realiza utilizando una membrana aniónica para evitar el gasto de $\mathrm{NaOH}$. El ion $\mathrm{OH}^{-}$se transporta hasta el anolito y evita la adición de $\mathrm{NaOH}$. Este método se descartó por la oclusión de la membrana, en su lado que enfrenta al cátodo por formación de precipitados de sales de calcio (carbonatos e hidróxidos).

Las Figura 13A y 13B, muestran un esquema de los métodos.

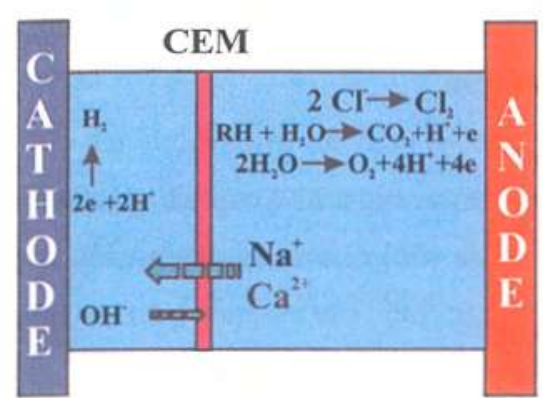

(A)

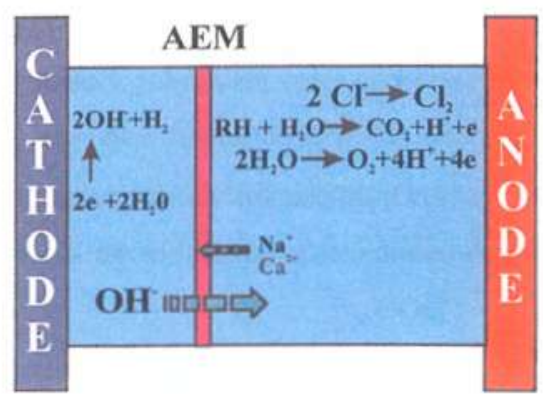

(B)
Figura 13. Métodos de electrólisis utilizados para el tratamiento del efluente industrial. (A) métodos 1 y 2, (B) método 3 .

\section{Resultados.}

Los resultados obtenidos fueron los siguientes:

1.- Concentración final de fenol inferior a $1 \mathrm{ppm}$.

2.- Disminución de la DQO entre el 70 y el $80 \%$ del valor inicial. Los valores finales están comprendidos entre 500-3000 ppm.

3.- No aparecen organoclorados de cadena $\mathrm{C} 2$ o superiores ni en la fase acuosa ni en la gaseosa.

4.- Se detecta cloroformo en la fase acuosa y gaseosa. La concentración en la fase acuosa oscila entre 1 y $25 \mathrm{ppm}$, dependiendo del método utilizado y del tiempo de electrólisis.

5.- En fase gaseosa los componentes mayoritarios son el oxígeno, nitrógeno y dióxido de carbono.

6.- Los sólidos en suspensión se mantienen por debajo del límite legal después del tratamiento.

7. La eficiencia del proceso electroquímico es similar usando los electrodos de dióxido de plomo o los de dióxido de plomo dopados con bismuto.

8.- El método 2 parece ser el más idóneo. Se consigue un mejor comportamiento en la eliminación de DQO y la minimización de los sólidos en suspensión. 
Sin embargo, para disminuir el coste eléctrico se ha llevado a cabo un proceso de optimización de estos resultados, basado en el esquema:

- Optimización de la densidad de corriente empleada con el objetivo de minimizar el coste energético y aumentar la eficiencia de la eliminación de la DQO.

- Elección óptima del material catódico para minimizar el coste energético.

- Determinación cuantitativa de cloroformo en la fase gaseosa. Si es necesario, elección del sistema más idóneo para su adsorción.

- Diseño de un proceso para la disminución de la concentración de cloroformo en la fase acuosa.

Los resultados obtenidos en este proceso de optimización han sido:

1.-Eliminación casi total de fenol, independientemente de la densidad de corriente usada (50-100 $\left.\mathrm{mAcm}^{-2}\right)$, con unos valores finales del orden de 0.1 ppm.

2.-Disminución de la DQO entre el $75-90 \%$ del valor inicial. Los valores finales oscilan entre 700-1200 ppm. Los mejores resultados se obtienen con densidades de corriente de $100 \mathrm{mAcm}^{-2}$.

3. La concentración de cloroformo en la fase gaseosa oscila entre 20 y 180 ppm, dependiendo de la densidad de corriente y del tiempo de tratamiento electrolítico

4. Si se mantiene la disolución final a $\mathrm{pH}$ básico, entre 11 y 12 , la concentración de cloroformo disminuye apreciablemente con el tiempo en la fase acuosa, obteniéndose una disminución de la concentración de cloroformo en un $75 \%$ aproximadamente.

5. Disminución del coste energético a valores no superiores a $1 \mathrm{pts} / \mathrm{dm}^{3}$ tratado para una disminución de $5000 \mathrm{ppm}$ en la DQO. Costes en el rango de 0.07 $0.16 \mathrm{kWh} / \mathrm{dm}^{3}$ dependiendo de la muestra.

$6 \mathrm{El}$ uso de cátodos tridimensionales de carbón y níquel hace disminuir el potencial necesario aunque el níquel presentó problemas de estabilidad.

7. El tamaño del reactor necesario oscila entre $1.1-2.4 \mathrm{~m}^{2} / \mathrm{m}^{3}$.día de efluente tratado para una disminución de 5000 ppm de DQO

\section{Estudio comparativo entre los electrodos $\mathrm{Ti} / \mathrm{Pt}$ y $\mathrm{Ti} / \mathrm{PbO}_{2}$}

Es dificil hoy en dia, encontrar empresas que comercialicen los electrodos $\mathrm{T} / \mathrm{PbO}_{2}$, de ahi que en el proceso de escalado a planta pre-piloto industrial nos hayamos visto obligados a sustituir el electrodo de dióxido de plomo por otro electrodo más comercial tal y como es el Ti/Pt [25].

En la Tabla 4 se muestran las condiciones del proceso para los electrodos Ti/Pt y $\mathrm{Ti} / \mathrm{PbO}_{2}$, mientras que los resultados se comparan en la Tabla 5.

\begin{tabular}{l}
\hline \multicolumn{1}{c}{ Experimental condtions } \\
\hline Anode $\mathrm{PbO}_{2} / \mathrm{Ti}$ or $\mathrm{Pt} / \mathrm{Ti}$ \\
Cathode Carbon felt. Catholyte $0.5 \mathrm{M}$ \\
$\mathrm{H}_{2} \mathrm{SO}_{4}$ \\
$\mathrm{Membrane} \mathrm{Nafion} 117$. Current $_{\text {density } 100 \mathrm{~mA} / \mathrm{cm}^{2} \text {. }}$ \\
Charge $150 \%$. Temperature $40^{\circ} \mathrm{C}$. \\
$63 \mathrm{~cm}^{2}$ geometric area. \\
\hline
\end{tabular}

Tabla 4. Condiciones experimentales correspondientes a las electrólisis sobre electrodos Ti'Pt y $\mathrm{Ti} / \mathrm{PbO}_{2}$

$\mathrm{PbO}_{2} / \mathrm{Ti}$

\begin{tabular}{c|cccll}
\hline $\begin{array}{c}\text { Sample } \\
\text { L09 }\end{array}$ & $\begin{array}{c}\mathrm{C}_{\text {phenol }} \\
\mathbf{p p m}\end{array}$ & $\begin{array}{c}\text { COD } \\
\text { Ppm }\end{array}$ & $\begin{array}{c}\text { TSS } \\
\mathbf{p p m}\end{array}$ & Gas phase & Aqueous phase \\
\hline $\begin{array}{c}\text { Initial } \\
\text { Anolyte }\end{array}$ & 21 & 3730 & -748 & & \\
\hline $\begin{array}{c}\text { Final } \\
\text { Anolyte }\end{array}$ & 0.03 & $\begin{array}{c}785 \\
79 \%\end{array}$ & 1414 & $\begin{array}{l}\mathrm{O}_{2}, \mathrm{~N}_{2}, \\
\mathrm{CO}_{2} \\
\mathrm{CHCl}_{3} \\
29.2 \mathrm{ppm}\end{array}$ & $\begin{array}{l}\text { No phenol, related } \\
\text { compounds or } \\
\text { halocompounds other } \\
\text { than } \mathrm{CHCl}_{3}(7.1 \mathrm{ppm})\end{array}$ \\
\hline $\begin{array}{c}\text { Final } \\
\text { Catholyte }\end{array}$ & & 74 & -- & & \\
\hline
\end{tabular}


Pt/Ti

\begin{tabular}{c|cccll}
\hline $\begin{array}{c}\text { Sample } \\
\text { L09 }\end{array}$ & $\begin{array}{c}\mathbf{C}_{\text {phenol }} \\
\text { ppm }\end{array}$ & $\begin{array}{c}\text { COD } \\
\text { ppm }\end{array}$ & $\begin{array}{c}\text { TSS } \\
\text { ppm }\end{array}$ & Gas phase & Aqueous phase \\
\hline $\begin{array}{c}\text { Initial } \\
\text { Anolyte }\end{array}$ & 21 & 3730 & -718 & & \\
\hline $\begin{array}{c}\text { Final } \\
\text { Anolyte }\end{array}$ & 0.06 & 860 & 1890 & $\begin{array}{l}\mathrm{O}_{2}, \mathrm{~N}_{2}, \\
\mathrm{CO}_{2} \\
\mathrm{CHCl}_{3} \\
87.7 \mathrm{ppm}\end{array}$ & $\begin{array}{l}\text { No phenol, related } \\
\text { compounds or } \\
\text { halocompounds other } \\
\text { than } \mathrm{CHCl}_{3}(2.5 \mathrm{ppm})\end{array}$ \\
\hline $\begin{array}{c}\text { Final } \\
\text { Catholyte }\end{array}$ & & 179 & -- & & \\
\hline
\end{tabular}

Tabla 5. Caracteristicas finales del efluente tratado electroquímicamente comparando el comportamiento de los electrodos de $\mathrm{Ti} / \mathrm{PbO}_{2}$ y $\mathrm{Ti} / \mathrm{Pt}$. TSS $=$ sólidos totales en suspensión.

\section{Proceso pre-piloto industrial.}

Para llevar a cabo el escalado a planta pre-piloto se ha empleado un reactor filtro prensa con un área unitaria de $200 \mathrm{~cm}^{2}$. El estudio realizado ha consistido en: i) Optimización de la densidad de corriente. ¿Constante o variable? ii) Comportamiento del electrodo con el tiempo, iii) Comportamiento de los separadores, iv) Estrategias para la eliminación de compuestos halogenados, v) Coste energético.

Se ha eliminado la membrana catiónica y sustituido por un separador de polietileno en un intento de disminuir los costes del reactor electroquímico.

Las condiciones experimentales han sido:

Ánodo: Titanio platinizado.

Cátodo: Acero inoxidable.

Separador: Polietileno.

Catolito: $0.5 \mathrm{M} \mathrm{NaOH}$

Densidad de corriente: $50-100 \mathrm{~mA} \mathrm{~cm}^{-2}$.

Reactor: Célula filtro-prensa $200 \mathrm{~cm}^{2}$ de área.

Tratamiento previo: Eliminación de $\mathrm{Ca}^{2+}$.
La presencia de ion $\mathrm{Ca}^{2+}$ en las aguas residuales origina la aparición, al cabo del tiempo, de depósitos de carbonatos sobre el electrodo que causan su rápida destrucción. Se produce también la oclusión del separador por lo que estos iones deben ser eliminados cambiado el proceso industrial o precipitándolos como carbonato cálcico antes de empezar el tratamiento.

En la tabla 6 se muestra las características más relevantes de algunos lotes industriales utilizados para este estudio mientras que la Tabla 7 da una idea de los costes eléctricos y tamaño del reactor necesario en diferentes condiciones del proceso.

\begin{tabular}{|c|c|c|c|c|c|}
\hline Analysis & $\begin{array}{c}\text { Sample } \\
\text { PPARF1 }\end{array}$ & $\begin{array}{c}\text { Sample } \\
\text { PPARF4 }\end{array}$ & $\begin{array}{c}\text { Sample } \\
\text { PPARF5 }\end{array}$ & $\begin{array}{c}\text { Sample } \\
\text { PPARF7 }\end{array}$ & $\begin{array}{c}\text { Sample } \\
\text { PPARF9 }\end{array}$ \\
\hline Phenol & 90 & 2 & 214 & 144 & 344 \\
\hline COD (ppm) & 2830 & 6690 & 9870 & 5090 & 12500 \\
\hline Conductivity $\left(\mathrm{mS} \mathrm{cm}^{-1}\right)$ & 11.2 & 11.8 & 11.8 & 12.8 & 12.5 \\
\hline Chloride (ppm) & 5620 & 5660 & 3475 & 2680 & 3680 \\
\hline Calcium (ppm) & 2650 & 2140 & 2720 & 1700 & 5800 \\
\hline
\end{tabular}

Tabla 6. Características iniciales más relevantes de muestras de efluentes empleados en el proceso pre-industrial.

\begin{tabular}{|c|c|c|c|c|c|c|}
\hline Experiment & $\begin{array}{l}\text { Electrodic } \\
\text { area } \\
\mathrm{m}^{2} / \mathrm{m}^{3} . \text { day } .5000 \\
\mathrm{ppm}\end{array}$ & $\begin{array}{l}\text { Electric cost } \\
\mathrm{kWh} / \mathrm{L} .5000 \\
\mathrm{ppm}\end{array}$ & $\begin{array}{l}\text { Phenol (ppm) } \\
\text { Final decrease \% }\end{array}$ & $\begin{array}{l}{ }^{C} \\
\text { final }\end{array}$ & $\begin{array}{l}\text { OD ppm } \\
\text { decrease \% }\end{array}$ & $\begin{array}{l}\text { Charge } \\
\text { passed } \\
\%\end{array}$ \\
\hline PPARF1001 & 1.58 & 0.33 & 99.8 & 960 & 66 & 150 \\
\hline PPARF4001 & 2.09 & 0.615 & 58 & 2800 & 50 & 150 \\
\hline PPARF5001 & 1.36 & 0.375 & 21 & 2230 & 77 & 150 \\
\hline *PPARF7001 & 2.91 & 0.43 & 100 & 1822 & 64 & 175 \\
\hline *PPARF9001 & 0.7 & 0.35 & 16 & 3470 & 72 & 150 \\
\hline
\end{tabular}

Tabla 7. Costes eléctricos y tamaño de reactor obtenidos de algunos experimentos realizados en el proceso pre-industrial. 
Se demuestra, pues, que es posible el tratamiento electroquímico de las aguas que contienen fenol a unos costes aceptables, aunque no bajos, y con un área electródica por $\mathrm{m}^{3}$ de efluente tratado no elevado.

\section{Referencias}

1] G. Sanchez-Cano, J.R.Perez, V. Montiel, A. Aldaz, Anales de Química, 85 (1989) 526.

[2] G. Sanchez-Cano, V. Montiel, A. Aldaz, Tetrahedron, 47(4/5) (1991) 877.

[3] G. Sanchez-Cano, G. Codina, J.R. Perez, v. Montiel, A. Aldaz, Ingeniería Quimica, (1992) 203.

[4] G. Sanchez-Cano, V. Montiel, V. Garcia, A. Aldaz, E. Elias, Electrochemical Engineering and Energy, Plenum Press, New York (1995)

[5] E. Expósito, J. González-García, V. García-García, V. Montiel, A. Aldaz, J. Electrochem. Soc,. 148 (3) (2001) 10.

[6] A. Aldaz, V. Montiel,G. Sanchez, M. López-Segura, N. Hernández, A. Planes, Spanish Patent ES 2,032,155, 1993.

[7] E. Elias, G. Sanchez-Cano, V.Montiel, V. Garcia-Garcia, A. Aldaz, Spanish Patent ES 2,061,397, 1994.

[8] E. Elias, G. Sanchez-Cano, V. Montiel, V. Garcia-Garcia, A. Aldaz. Spanish Patent, P9300403, 1993

[9] V. Garcia, G. Sanchez-Cano, V. Montiel, E. Elias, A. Ginebreda, A. Aldaz. Eur Pat. Appl. EP 618,312, 1994.

[10]A. Aldaz, F. Carmona, M. Esteban, V.Garcia Garcia, J. González Garcia, V. Montiel, G. Sanchez, ES9501098, 1995, PCT/ES96/00122, 1996.

[11]Aldaz Riera Antonio, Montiel Leguey Vicente, García García Vicente, José González García. ES601014, 1996. PCT/ES97/00113 WO 97/42358, 1997. Int Cl C25B 04, Fecha de publicación 26/01/2000 con nº 97919417.2

[12]V. Montiel, XX Reunión del Grupo Especializado de Electroquímica y IV Reunión Ibérica de Electroquímica. Universidad de Alicante, 1997

[13]V. Montiel, Workshop on Electrochemistry Applied to Industry and Environmental protection, Universidad de Alicante, 1997
[14]A. Aldaz, V. Montiel, J. González, V.García-García, $11^{\text {th }}$ International Forum Electrolysis in the Chemical Industry. Electrochemical Processing Technologies. Miami, Florida, USA, 1997

[15] J. González-García, V. García-García, V. Montiel, A. Aldaz. European Research Conference: "Organic Electrochemistry: Moving Towards Clean and Selective Synthesis", Agelonde-La Londe, France, 1998.

[16] J. González-García, V. García-García, V. Montiel, G. Sánchez-Cano, A. Aldaz, The $195^{\text {th }}$ Meeting of the Electrochemical Society, Seattle, 1999.

[17] T. R. Ralph, M. L. Hitchman, J. P. Millington, F. Walsh, J. Electroanal. Chem., 375 (1994) 17.

[18] J. González-García, V. Garcia-Garcia, V. Montiel, A. Aldaz, $13^{\text {th }}$ Forum Electrolysis in the Industry, Miami, 1999.

[19] J. González-García, V. Montiel, G. Sanchez-Cano, A. Aldaz, Spanish Patent, 9401259, (1994)

[20] J. Iniesta, Tesis Doctoral, Universidad de Alicante, (1999).

[21] J. Iniesta, J. González-García, V. García-García, V. Montiel, A.Aldaz, Ind. Eng. Chem. Res., submitted.

[22] W. R. La Course, Y.-L. Hsian, D. C. Johnson, W.H. Weber, J. Electrochem. Soc., 136 (1989) 3714.

[23]J. Iniesta, E. Expósito, J. González-García, V. Montiel, A. Aldaz, Water Res., in press.

[24] J. González-García, J.A. Conesa, J. Iniesta, V. García-García, V. Montiel, A. Aldaz, J. Chem. E. Symp. Ser. 145, Proceedings of the fifth European Symposium of Electrochemical Engineering, ed. AA. Wragg, p51, Exeter, Amsterdam.

[25] J. Iniesta, J. González-García, J. Fernández, V. Montiel, A. Aldaz, J. Mater Chem, 9 (1999) 3141. 